\title{
Importance of Time Scale and Local Environment in Electron-Driven Proton Transfer. The Anion of Acetoacetic Acid
}

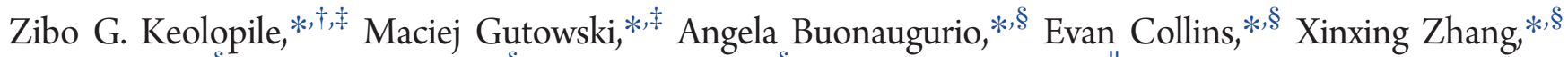 \\ Jeremy Erb, ${ }^{*, \S}$ Thomas Lectka, ${ }^{*} \S$ Kit H. Bowen, ${ }^{*}$, and Michael Allan ${ }^{*, \|}$ \\ ${ }^{\dagger}$ Department of Physics, University of Botswana, Private Bag 0022, Gaborone, Botswana \\ ${ }^{\ddagger}$ Institute of Chemical Sciences, School of Engineering and Physical Sciences, Heriot-Watt University, Edinburgh EH14 4AS, United \\ Kingdom \\ ${ }^{\S}$ Department of Chemistry, Johns Hopkins University, Baltimore, Maryland 21218, United States \\ "Department of Chemistry, University of Fribourg, chemin du Musée 9, Fribourg 1700, Switzerland
}

\begin{abstract}
Anion photoelectron spectroscopy (PES) and electron energy-loss spectroscopy (EELS) probe different regions of the anionic potential energy surface. These complementary techniques provided information about anionic states of acetoacetic acid (AA). Electronic structure calculations facilitated the identification of the most stable tautomers and conformers for both neutral and anionic AA and

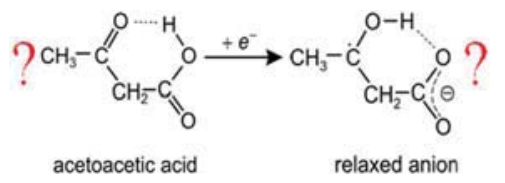
determined their relative stabilities and excess electron binding energies. The most stable conformers of the neutral keto and enol tautomers differ by less than $1 \mathrm{kcal} / \mathrm{mol}$ in terms of electronic energies corrected for zero-point vibrations. Thermal effects favor these conformers of the keto tautomer, which do not support an intramolecular hydrogen bond between the keto and the carboxylic groups. The valence anion displays a distinct minimum which results from proton transfer from the carboxylic to the keto group; thus, we name it an ol structure. The minimum is characterized by a short intramolecular hydrogen bond, a significant electron vertical detachment energy of $2.38 \mathrm{eV}$, but a modest adiabatic electron affinity of $0.33 \mathrm{eV}$. The valence anion was identified in the anion PES experiments, and the measured electron vertical detachment energy of $2.30 \mathrm{eV}$ is in good agreement with our computational prediction. We conclude that binding an excess electron in a $\pi^{*}$ valence orbital changes the localization of a proton in the fully relaxed structure of the $\mathrm{AA}^{-}$anion. The results of EELS experiments do not provide evidence for an ultrarapid proton transfer in the lowest $\pi^{*}$ resonance of $\mathrm{AA}^{-}$, which would be capable of competing with electron autodetachment. This observation is consistent with our computational results, indicating that major gas-phase conformers and tautomers of neutral AA do not support the intramolecular hydrogen bond that would facilitate ultrarapid proton transfer and formation of the ol valence anion. This is confirmed by our vibrational EELS spectrum. Anions formed by vertical electron attachment to dominant neutrals undergo electron autodetachment with or without vibrational excitations but are unable to relax to the ol structure on a time scale fast enough to compete with autodetachment.
\end{abstract}

\section{INTRODUCTION}

Proton motion coupled with electron transfer was identified long ago as the basic mechanism of bioenergetic conversion. ${ }^{1}$ An extensive study of this class of reactions has recently been motivated by their importance in biological systems, e.g., damage of DNA and RNA by low-energy electrons involved in cancer radiotherapy, ${ }^{2,3}$ protein redox machines including photosystem II and ribonucleotide reductase. ${ }^{4}$ There is also technological relevance in conversion and storage of solar energy through activation of small molecules such as water, methanol, and $\mathrm{CO}_{2} .{ }^{5}$ Finally, fabrication of micro- and nanoelectromechanical devices through electron beam lithography hinges on fundamental electron-driven processes. ${ }^{6} \mathrm{~A}$ special subcategory of proton-coupled electron transfer is excess electron-induced proton transfer. ${ }^{3,7}$

Although gas-phase studies of excess electron-induced proton transfer in DNA and proteins are challenging because of the low vapor pressures of these molecules, 3,8 this difficulty has been significantly reduced in the cases of subunits of DNA, e.g., base pairs, nucleosides, and nucleotides, by the development of specialized, laser desorption/photoemission anion sources for bringing them into the gas phase as intact anions. ${ }^{9-11}$ Nevertheless, it is still valuable to identify smaller model systems with greater vapor pressures, so that the fundamental chemical and physical aspects of (excess) electron-induced proton transfer can be studied.

Previously, we suggested that the dimer of formic acid might serve as a model system for intermolecular proton transfer induced by a $\pi^{*}$ excess electron. ${ }^{12}$ It displays many similarities with intermolecular proton transfer in anionic complexes of nucleic acid bases with weak acids. ${ }^{13-15}$ Similarly, hydrogenbonded complexes of ammonia and hydrogen halides served as model systems for intermolecular proton transfer induced by a $\sigma$ excess electron. ${ }^{16,17}$ 


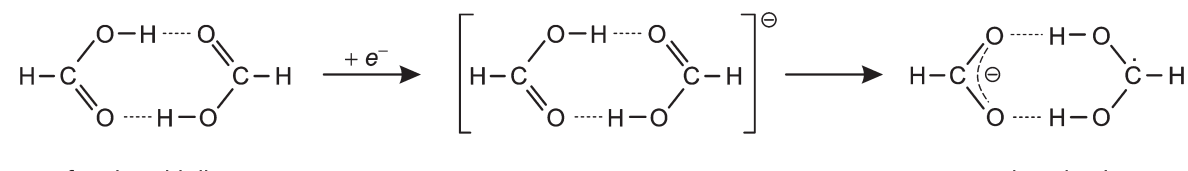

formic acid dimer

resonance

relaxed anion<smiles>CC(=O)COC(=O)CO</smiles>

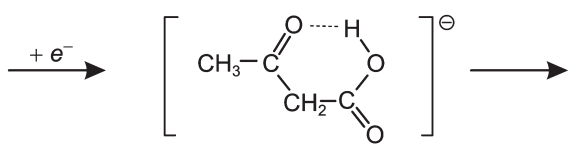

resonance

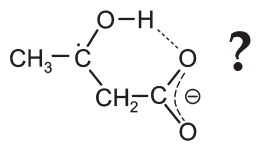

relaxed anion

Figure 1. Electron-induced intermolecular proton transfer in the formic acid dimer ${ }^{12,18,19}$ (top), and the hypothesized intramolecular proton transfer in the keto tautomer of acetoacetic acid (bottom). The feasibility of the latter process will be explored in this study for the tautomers of acetoacetic acid. On the basis of the formic acid results ${ }^{18}$ we expect that the vertical electron attachment leads to a (shape) resonance, with the excess electron in the lowest $\pi^{*}$ orbital, which can then relax by an ultrarapid proton transfer, in competition with the very fast spontaneous electron detachment.

The electron-induced proton transfer in the formic acid dimer (Figure 1, top row) is manifested also by the differences of the results of electron energy-loss spectroscopy (EELS) experiments on the monomer and dimer of formic acid. ${ }^{18}$ The yield of very low energy electrons was found to be 20 times stronger in the dimer than in the monomer. The dramatic increase in the efficiency of the dimer to quasi-thermalize electrons arriving in the $1-2 \mathrm{eV}$ energy range and captured in the lowest $\pi^{*}$ shape resonance was interpreted in terms of rapid intermolecular proton transfer that quenches the fast autodetachment channel. It was concluded that the phenomenon of electron-driven proton transfer can be ubiquitous and that it may be responsible for rapid slowing down of excess electrons.

The relaxed anion of the formic acid dimer has now been experimentally characterized using Ar-tagged vibrational predissociation and electron autodetachment spectroscopies as well as anion photoelectron spectroscopy. ${ }^{19}$ These results confirmed that excess electron attachment leads to a transfer of one of the protons across the H-bonded bridge. The study corroborated that the relaxed anion of formic acid dimer is composed of a largely intact formate anion attached to the dihydroxymethyl radical through a symmetrical, double $\mathrm{O}-\mathrm{H}$ bonded bridge, see the top of Figure 1.

Much less information is available on intramolecular proton transfer induced by an excess electron, and the results are available primarily for low-vapor pressure molecules, e.g., nucleotides. ${ }^{20}$ For example, the anion photoelectron spectrum of $2^{\prime}$-deoxyadenosine-5'-monophosphate ${ }^{20}$ has been interpreted through intramolecular proton transfer from a hydroxyl group of the phosphate to the $\mathrm{N} 3$ position of the adenine. ${ }^{21}$

Here, we present computational and experimental results on neutral and anionic acetoacetic acid (AA), see Figure 1 (bottom row). Acetoacetic acid is the simplest beta-keto acid and is thermodynamically unstable with respect to the decomposition to acetone and $\mathrm{CO}_{2}$. However, an experimental half-life of 140 min has been reported for a water solution of AA at $37{ }^{\circ} \mathrm{C}$. ${ }^{22}$ Thus, the molecule can be probed experimentally provided care is taken to operate at low temperatures. Some computational information is available on the ketonic decarboxylation of AA. $^{23-25}$

AA can exist in both the keto and the enol forms (labeled $\mathbf{K}$ and $\mathbf{E}$ here). ${ }^{25,26}$ The keto-enol equilibrium was found to be strongly solvent dependent, with the keto form dominant in polar solvents. On the basis of ${ }^{1} \mathrm{H}$ NMR spectra it was suggested that a conformer of the keto form with an intramolecular hydrogen bond ( $\mathbf{K} \mathbf{1}$ in Figure 2) is not
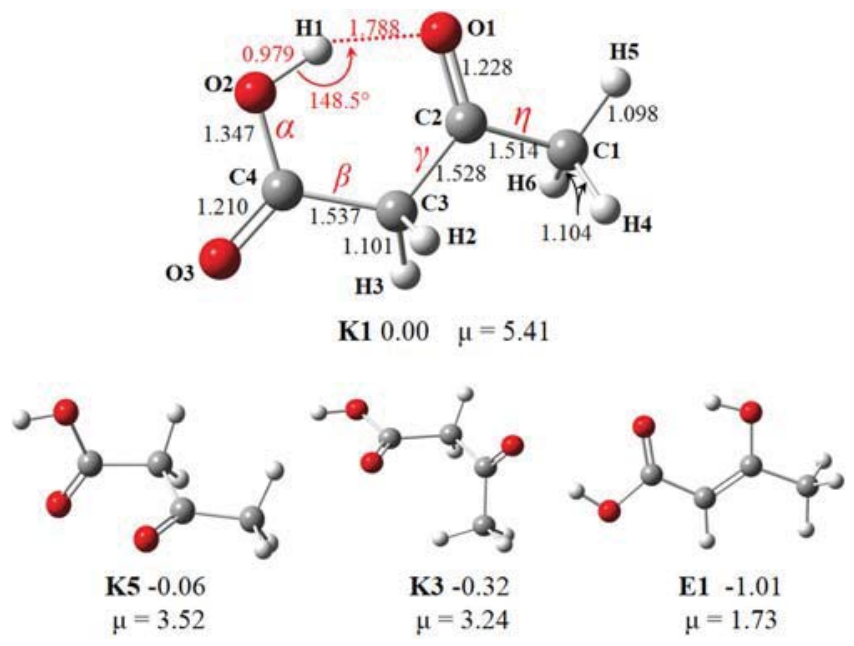

Figure 2. Molecular structures of neutral AA considered in this study. The relative $\mathrm{CCSD}(\mathrm{T}) / \mathrm{ADZ}$ energies corrected for $\mathrm{MP} 2 / \mathrm{ADZ}$ zeropoint energies $(\mathrm{kcal} / \mathrm{mol})$. Principal geometrical parameters (in Angstroms and degrees) were characterized at the CCSD/ADZ+DF level of theory. Bonds $\alpha, \beta, \gamma, \eta$, and $\varepsilon$ were rotated for conformational searches. Dipole moments, $\mu$, determined at the CCSD level, are in Debyes.

dominant under any conditions, though keto tautomers dominate in polar solvents. ${ }^{26}$ It was also suggested that an enol tautomer is present in less polar solvents, and it certainly exists as an internally hydrogen-bonded conformer, like E1. The enolization of the ketone group was found to be more favorable than the enolization of the carboxylic group by 11.3 $\mathrm{kcal} / \mathrm{mol}$ at the MP2 level of theory. ${ }^{25}$ Thus, our further discussion is focused on the keto and ketone enol tautomers, see Figure 2.

In this study, we explore $\mathrm{AA}$ in the gas phase and probe whether its keto and enol tautomers undergo intramolecular proton transfer driven by an excess electron attachment (Figures 1 (bottom) and 3). Upon formation of a suitable conformer, an intramolecular hydrogen bond might provide a bridge for ultrafast proton transfer. We use electronic structure methods to identify the most stable conformers of the keto and enol tautomers and to characterize the bound anionic states of AA, both valence and dipole bound. Anion photoelectron spectroscopy (PES) measurements probe the bound and relaxed anionic states of $\mathrm{AA}^{-}$and test computational predictions. EELS measurements provide both vertical electron attachment energies for the (unrelaxed) $\pi^{*}$ states (resonances) 


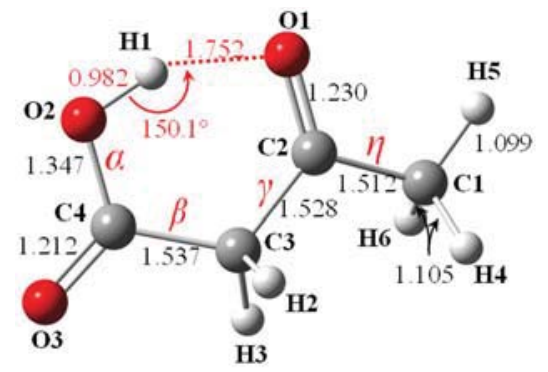

DB

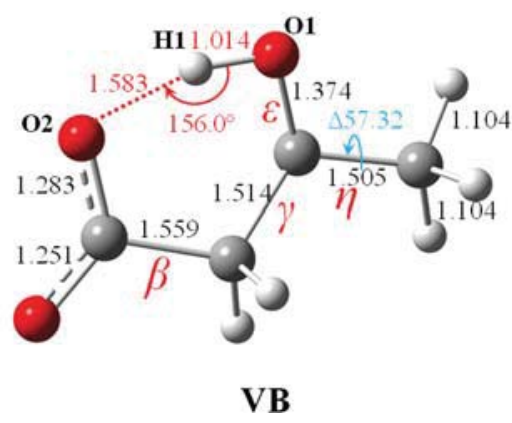

Figure 3. Molecular structures of valence-bound $(\mathbf{V B})$ and dipole-bound (DB) anions of AA, considered in this study. Principal geometrical parameters (in Angstroms and degrees) were characterized at the CCSD/ADZ+DF level of theory. Bonds $\alpha, \beta, \gamma, \eta$, and $\varepsilon$ were rotated for conformational searches. The change, $\Delta$, in the $\mathrm{O} 1-\mathrm{C} 2-\mathrm{C} 1-\mathrm{H} 5$ dihedral angle (blue) from the neutral $\mathrm{K} 1$ to the valence anion geometry is in degrees.

of $\mathrm{AA}^{-}$and the vibrational spectrum of neutral $\mathrm{AA}$. On the basis of past experience with the dimer of formic acid, ${ }^{18}$ particular attention is paid to the possibility of quasithermalization of electrons captured in the lowest $\pi^{*}$ shape resonance, indicative of an ultrarapid proton transfer.

\section{COMPUTATIONAL METHODS}

The keto and enol tautomers and conformers of neutral AA as well as the valence-bound anion (Figures 2 and 3) were explored using our inhouse potential energy surface scanning tool (PESST). ${ }^{29}$ When probing the conformational space of keto, the bond $\alpha$ was rotated with a step size of $180^{\circ}$. The bonds $\beta$ and $\gamma$ were rotated with a step size of $60^{\circ}$. The probed initial $\eta$ values were $0^{\circ}$ and $60^{\circ}$. All combinations of these rotatable bonds $\alpha, \beta, \gamma$, and $\eta$ resulted in 144 initial structures for screening purposes. Similarly, when probing the conformational space of enol, the bond $\gamma$ was rotated with a step size of $60^{\circ}$. The bonds $\beta, \alpha$, and $\varepsilon$ were rotated with a step size of $180^{\circ}$. All combinations of these rotatable bonds resulted in 48 initial structures for screening purposes. The valence anion of AA favors the ol structure. When probing its conformational space, $\gamma$ and $\varepsilon$ were rotated with a step size of $60^{\circ}$ and $180^{\circ}$, respectively. The initial $\beta$ values were $0^{\circ}$ and $90^{\circ}$, while those for $\eta$ were $0^{\circ}$ and $60^{\circ}$, yielding 42 initial structures.

We used the standard Dunning's aug-cc-pVDZ $(\mathrm{ADZ})^{28,29}$ basis set supplemented with extra low-exponent basis functions centered on the carbon of $\left(-\mathrm{CH}_{3}\right)$ to describe the diffuse charge distribution in the anionic dipole-bound state. The exponents $\alpha_{n}$ of these basis functions were determined through $\alpha_{n}=\alpha_{0} / q^{n}, n=1,2, \ldots$, initiated from the lowest exponent $\alpha_{0}$ of the $s, p$, or $d$ functions in the standard ADZ basis set and advanced with $q=3.2{ }^{30}$ We limited the extra diffuse set to $5 s, 5 p$, and $2 d$ functions. ${ }^{31,32}$ We will use a label DF for these additional diffuse functions and $\mathrm{ADZ}+\mathrm{DF}$ for the combined basis set.

The initial structures were prescreened at the density functional level of theory with the B3LYP exchange-correlation functional. ${ }^{33-36}$ The minima contained within an energy range of $10 \mathrm{kcal} / \mathrm{mol}$ were reoptimized at the coupled-cluster singles and doubles (CCSD) ${ }^{37}$ level of theory. Harmonic frequencies were routinely calculated at the MP2 level and for the most stable systems also at the CCSD level. The most accurate electronic energies were obtained at the coupled-cluster level with single, double, and noniterative triple excitations $(\operatorname{CCSD}(\mathrm{T})) .{ }^{38}$ The Gibbs energies were based on the coupled cluster electronic energies corrected for zero-point vibration energies, thermal contributions to energy, $\mathrm{pV}$ terms, and entropy terms. These terms were calculated in the rigid rotor-harmonic oscillator approximation for $T=298 \mathrm{~K}$ and $p=1 \mathrm{~atm}$. We also characterized barriers separating low-lying conformers of the neutral keto AA.

The vertical excess electron binding energies were calculated in two ways. In "indirect" approaches, the energy of the anion was subtracted from the energy of the neutral, and the procedure was executed at the SCF, MP2, CCSD, and CCSD $(T)$ levels of theory. The vertical excess electron binding energy can be also calculated "directly". Here we used the electron propagator theory (EPT) method with the P3 propagator $^{39}$ applicable to both electron detachment and attachment processes. We will use the term EPT(third) to refer to the third-order electron binding energies. One can calculate the excess electron vertical binding energy as either the electron affinity (EA) of the neutral or the ionization potential (IP) of the anion.

The dipole- and valence-bound anions have minima corresponding to quite different molecular structures. The former results from a minor distortion of the neutral K1 keto, but the latter is better described as a conformer of the ol tautomer (Figure 3). Henceforth we will use DB and VB to refer to the dipole- and valence-bound anions, respectively. In order to illustrate the evolution of the DB and VB anionic states as a function of intramolecular proton transfer, we constructed a linear synchronous path, see eq S1 in the Supporting Information.

The energy of the anion $M^{-}$at a geometry $G$ can be written as

$$
E_{M^{-}}(G)=E_{M}\left(G_{M}\right)+\Delta E_{M}(G)-E B E(G)
$$

where $E_{M}\left(G_{M}\right)$ is the energy of the neutral $M$ at its optimal geometry $G_{M}$

$$
\Delta E_{M}(G)=E_{M}(G)-E_{M}\left(G_{M}\right)
$$

represents an increase of the energy of the neutral $M$ associated with its geometrical deformation from $G_{M}$ to $G$, and $\operatorname{EBE}(G)$ is the vertical electron binding energy at the geometry $G$

$$
\operatorname{EBE}(G)=E_{M}(G)-E_{M^{-}}(G)
$$

The values of $E B E$ are positive for vertically bound anionic states and negative for resonances. The vertical electron affinity (VEA) is equivalent to $\operatorname{EBE}\left(G_{M}\right)$. The vertical detachment energy (VDE) is equivalent to $\operatorname{EBE}\left(G_{M^{-}}\right)$, and the electronic part of the adiabatic electron affinity is defined as

$$
\mathrm{AEA}=E_{M}\left(G_{M}\right)-E_{M^{-}}\left(G_{M^{-}}\right)
$$

where $G_{M^{-}}$is the optimal geometry of the anion of $M$. Notice that

$$
\mathrm{AEA}=-\Delta E_{M}\left(G_{M^{-}}\right)+\operatorname{EBE}\left(G_{M^{-}}\right)=-\Delta E_{M}\left(G_{M^{-}}\right)+\operatorname{VDE}
$$

Further extensions of this notation are needed for $G_{M^{-}}$, as the anion might be either valence or dipole bound. In addition to $G_{M}$ we will consider $G_{M_{\mathrm{dbs}}^{-}}$and $G_{M_{\mathrm{Vv}}^{-}}$, which are the lowest energy structures for the dipole- and valence-bound anions, respectively. We will also consider a quantity

$$
\Delta E_{\mathrm{VB} \rightarrow \mathrm{dbs}}=E_{M_{\mathrm{dbs}}^{-}}\left(G_{M_{\mathrm{VB}}^{-}}\right)-E_{M_{\mathrm{VB}}^{-}}\left(G_{M_{\mathrm{VB}}^{-}}\right)
$$

which is the vertical excitation energy from the valence- to the dipolebound anion at the optimal valence anion geometry.

All electronic structure calculations reported in this study were performed with the Gaussian 2009 package. ${ }^{40}$ The orbitals occupied by an excess electron were generated with the Visual Molecular Dynamics ${ }^{41}$ package, and the contour values used in the plots were 
calculated with the OpenCubeMan ${ }^{42}$ tool using a fraction of electron $\left(F_{\mathrm{e}}\right)$ density equal to 0.6 . The GaussView ${ }^{43}$ package was used to draw molecular structures.

\section{EXPERIMENTAL METHODS}

3.1. Synthesis of Acetoacetic Acid. Our synthesis followed the protocol described in ref 44 . Sodium hydroxide $(0.16 \mathrm{~mol})$ was added to aqueous ethyl acetoacetate $(0.15 \mathrm{~mol})$ on ice. The reaction was stirred and allowed to react overnight. The resulting solution was saturated with ammonium sulfate. Sulfuric acid was then used to acidify the solution. The solution was extracted three times with diethyl ether $(200 \mathrm{~mL})$ and dried over magnesium sulfate. After removing the ether using a rotary evaporator and drying using a rotary vacuum pump, a yellowish gel of acetoacetic acid was obtained. Since batches of acetoacetic acid were readily subject to decomposition, care was taken to ensure that the solutions did not become warmer than 30 ${ }^{\circ} \mathrm{C}$, and they were utilized in PES and EELS experiments as quickly as possible.

3.2. Photoelectron Spectroscopy. Anions of acetoacetic acid were generated by two different sources and their photoelectron spectra measured on two different types of anion photoelectron spectrometers. In one case, they were produced with a nozzle-ion source and their photoelectron spectra measured with a continuous anion photoelectron apparatus. ${ }^{45}$ In the other case, the anions were generated with a photoinduced electron emission source, and their photoelectron spectra were measured with a pulsed anion photoelectron apparatus. ${ }^{46}$ In both instruments, anion photoelectron spectroscopy was conducted by crossing a mass-selected beam of negative ions with a fixed-frequency photon beam and then energy analyzing the resultant photodetached electrons. This technique is governed by the energy conserving relationship $h v=E K E+E B E$, where $h v$ is the photon energy, EKE is the measured electron kinetic energy, and $E B E$ is the electron binding energy.

3.2.1. Continuous Anion Photoelectron Spectrometer. In this type of anion photoelectron spectrometer, the ion source and all of the other components operate continuously. ${ }^{45}$ The ion source was a biased $(-500 \mathrm{~V})$, supersonic expansion nozzle-ion source in which the acetoacetic acid sample was placed inside its stagnation chamber. There, due to the warming of the source by the adjacent hot filament, some of the sample evaporated and was expanded through a $25 \mu \mathrm{m}$ diameter nozzle orifice into $\sim 10^{-4}$ Torr vacuum along with argon gas which was maintained at a pressure of $1-2 \mathrm{~atm}$ in the source's stagnation chamber. Anions were formed by injecting low-energy electrons from a hot, even more negatively biased, thoriated iridium filament into the expanding jet, where a weak external magnetic field helped to form a microplasma. The nascent anions were then extracted into ion optics and mass selected by a $90^{\circ}$ magnetic sector, mass spectrometer with a mass resolution of $\sim 400$. After mass selection, the beam of acetoacetic acid parent anions was crossed with an argon ion laser beam (operated intracavity), where electrons were photodetached. These were then energy analyzed by a hemispherical electron energy analyzer operating at a resolution of $30 \mathrm{meV}$. The photoelectron spectra reported here were recorded with $2.54 \mathrm{eV}$ photons and calibrated against the well-known photoelectron spectrum of $\mathrm{O}^{-}$.

3.2.2. Pulsed Anion Photoelectron Spectrometer. In this type of anion photoelectron spectrometer the ion source and all of the other components operate in a pulsed manner. ${ }^{46}$ Anions were generated by the interaction of laser-generated photoelectrons with a pulsed jet of helium gas containing a small amount of acetoacetic acid vapor. The photoelectrons were produced by pulsed laser irradiation (Nd:YAG laser operating at $2.33 \mathrm{eV} /$ photon) of a rotating, translating copper rod which was mounted inside a grounded housing having a laser beam entrance port, a pulsed gas valve, and an exit nozzle. A small amount of acetoacetic acid sample was placed inside the pulsed gas valve, and together with $4 \mathrm{~atm}$ of helium gas, its vapor was expanded in synchronization with the laser pulses. Photoemitted electrons attached to $\mathrm{AA}$ molecules to form $\mathrm{AA}^{-}$anions. These were entrained in the ensuing jet which was directed through a skimmer and a subsequent differential pumping chamber into the Wiley-McLaren extraction plates of a linear, time-of-flight mass spectrometer (mass resolution 500). After mass selection by a mass gate and deceleration via a momentum decelerator, the anions of interest were irradiated by a second pulsed laser beam (Nd:YAG laser operating at $3.49 \mathrm{eV} /$ photon), which photodetached electrons from them. The photodetached electrons were then energy analyzed by a magnetic bottle, electron energy analyzer with a resolution of $35 \mathrm{meV}$ at $E K E=1 \mathrm{eV}$. The photoelectron spectrum was calibrated against the well-known atomic lines of $\mathrm{Cu}^{-48}$ The pulsed photoelectron spectrometer probed a wider range of electron binding energies than the continuous photoelectron spectrometer due to its use of higher energy photons.

3.3. Electron Energy-Loss Spectroscopy. The electron-impact spectrometer used in this study has been described in the work on the formic acid $\operatorname{dimer}^{18}$ (and references cited therein). It uses hemispherical analyzers to prepare a beam with a narrow electron energy spread and to analyze energies of the scattered electrons. The measurements were performed at a scattering angle of $135^{\circ}$. The sample was kept in ice and evaporated through a needle valve to a 0.25 $\mathrm{mm}$ diameter effusive orifice kept at $30^{\circ} \mathrm{C}$. The resolution was about $20 \mathrm{meV}$ in the energy-loss mode.

\section{COMPUTATIONAL RESULTS}

4.1. Neutral AA. Neutral AA supports minima for the keto and enol tautomers (Figure 2). Our attempts to identify an ol minimum energy structure with $\mathrm{H} 1$ bound to $\mathrm{O} 1$ failed: the $\mathrm{H} 1$ proton either transferred back to $\mathrm{O} 2$ or the molecule broke into propen-2-ol and $\mathrm{CO}_{2}{ }^{23}$ There are six low-lying keto conformers $(\mathrm{K} 1-\mathrm{K} 6)$ within an energy range of $1 \mathrm{kcal} / \mathrm{mol}$. Their structures and the transition states separating them are presented in the Supporting Information, Figure S1. The enol conformers are presented there as well in Figure S2. The most stable E1 is separated from other enols by at least $4 \mathrm{kcal} / \mathrm{mol}$. The minima of the neutral AA relevant for the further discussion (K1, K3, K5, E1) are presented in Figure 2.

The relative stability of $\mathbf{K} \mathbf{1}$ with respect to $\mathbf{K} \mathbf{3}, \mathbf{K 5}$, and $\mathbf{E} \mathbf{1}$ is summarized in Table 1. In terms of electronic energies, the enol

Table 1. Relative Stability ( $\mathrm{kcal} / \mathrm{mol}$ ) of the Most Relevant Tautomers and Conformers of Neutral AA

\begin{tabular}{lcrccc} 
str. & $E_{\text {elec }}^{\mathrm{MP2}}$ & \multicolumn{1}{c}{$E_{\text {elec }}^{\mathrm{CCSD}}$} & \multicolumn{1}{c}{$E_{\text {elec }}^{\mathrm{CCSD}(\mathrm{T})}$} & $E_{\text {elect }}^{\mathrm{CCSD}(\mathrm{T})}+E_{0, \mathrm{vib}}^{\mathrm{MP2}}$ & $E_{\text {elec }}^{\mathrm{CCSD}(\mathrm{T})}+$ Gibbs \\
$\mathrm{K} 1$ & 0.00 & 0.00 & 0.00 & 0.00 & 0.00 \\
$\mathrm{~K} 3$ & 0.04 & -0.41 & 0.01 & -0.32 & -0.98 \\
$\mathrm{~K} 5$ & 0.24 & -0.13 & 0.28 & -0.06 & -0.82 \\
$\mathrm{E} 1$ & -2.85 & -1.55 & -1.91 & -1.01 & -0.20 \\
\hline
\end{tabular}

E1 is the most stable at all levels of theory by ca. $2 \mathrm{kcal} / \mathrm{mol}$. The keto conformers are clustered within a narrow range of 0.3 $\mathrm{kcal} / \mathrm{mol}$, with $\mathrm{K} \mathbf{1}$ being the most stable. With such small differences in electronic energies, the zero-point energies and thermal contributions to thermodynamic functions clearly matter. As was observed in the past, conformers with hydrogen bonds have higher zero-point vibrational energies despite significant red shifts of the proton donor stretching frequencies. ${ }^{49,50}$ The same pattern is observed here, i.e., E1 and $\mathrm{K} 1$ have the highest zero-point vibrational corrections in the enol and keto families, respectively. The thermal contribution to the stability is dominated by entropic effects associated with very soft vibrational modes. Thus, again E1 and K1 are disfavored, and the most stable gaseous conformers at standard conditions are $\mathbf{K} \mathbf{3}$ and $\mathbf{K 5}$, though the spread of Gibbs free energies is very narrow, less than $1 \mathrm{kcal} / \mathrm{mol}$. Our findings are consistent with the earlier liquid-phase ${ }^{1} \mathrm{H}$ NMR results of Grande and Rosenfeld. ${ }^{26}$ 
A simplified landscape of the potential energy surface for the keto conformers is illustrated in Figure 4. One could anticipate

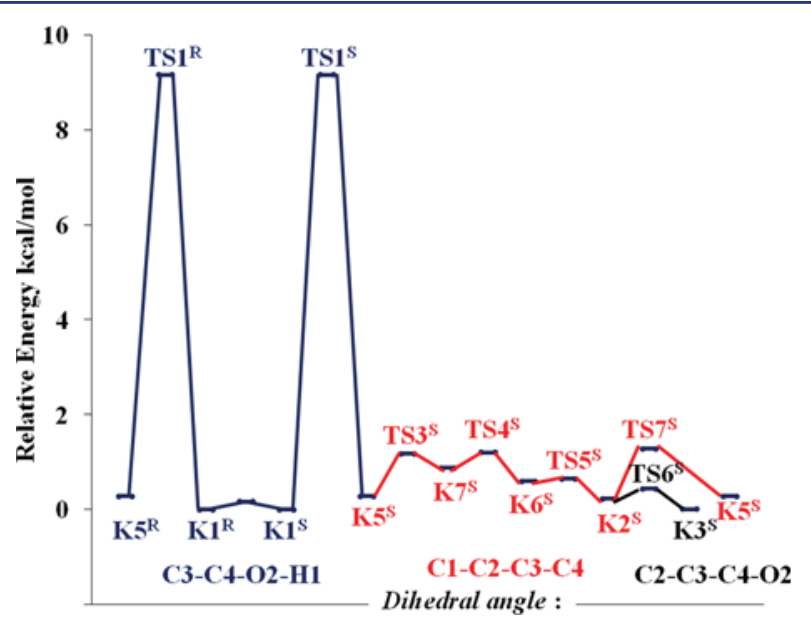

Figure 4. Energy profile connecting keto structures of AA. Energies $(\mathrm{kcal} / \mathrm{mol})$ were calculated at the $\operatorname{CCSD}(\mathrm{T}) / \mathrm{ADZ}$ level using the $\mathrm{CCSD} / \mathrm{ADZ}$ geometries.

that the $\mathbf{K} \mathbf{1}$ conformer would be particularly stable due to the intramolecular $\mathrm{O} 2 \mathrm{H} 1 \cdots \mathrm{O} 1$ hydrogen bond, but the relative energies listed in Table 1 and Figure S1 indicate otherwise. K1 is unique not so much in its stability but in the energy barrier (TS1 at $9.2 \mathrm{kcal} / \mathrm{mol}$ ) that separates it from $\mathbf{K 5}$ and the remaining conformers. This barrier can be associated with breaking of the intramolecular hydrogen bond. The barriers separating the conformers $\mathbf{K} \mathbf{2}-\mathbf{K} 7$ are much smaller and do not exceed $1.1 \mathrm{kcal} / \mathrm{mol}$.

There are at least two factors that oppose the stabilizing effect of the intramolecular hydrogen bond in K1. First, K1 is the most polar conformer, with a dipole moment exceeding 5.4 D. The remaining conformers have dipole moments smaller by more than $1.8 \mathrm{D}$. High polarity typically increases the electronic energy of a neutral molecule by raising the energy of the highest occupied orbital. Second, formation of the $\mathrm{O} 2 \mathrm{H} 1 \cdots \mathrm{O} 1$ hydrogen bond is associated with intramolecular strain. The results obtained with the Amber ${ }^{51}$ force field, see Table S5, confirm this hypothesis. A sum of the angle bending, torsional and bond stretching terms is larger in K1 than in, e.g., K5, by ca. $2.8 \mathrm{kcal} / \mathrm{mol}$.

4.2. Anionic AA. $\mathrm{K} 1$ is the most promising conformer to host a dipole-bound anionic state in view of its competitive stability and dominant polarity $(\mu=5.4 \mathrm{D})$. In addition to the dipole-bound state, AA can support a valence-bound anion. An overview of anionic states of AA is presented in Figure 5 using a set of geometries connecting the dipole-bound minimum, similar to the K1 structure of the neutral, with the valencebound anion minimum: $G_{M_{\mathrm{dbs}}^{-}}$with $G_{M_{\mathrm{VB}}^{-}}$. The potential energy curve of the neutral is repulsive upon transferring $\mathrm{H} 1$ from $\mathrm{O} 2$ to $\mathrm{O}$. The dipole moment of the neutral increases upon proton transfer from 5.4 to $10.0 \mathrm{D}$. Thus, the dipole-bound anion remains bound upon proton transfer. The valence anion is unbound at the minimum energy structure of the neutral; it can be probed as a resonance state with a very short finite lifetime in the EELS experiments (see section 5.2). The energy of the resonance quickly decreases upon intramolecular proton transfer, crosses the potential energy surface of the neutral, and undergoes an avoided crossing with the dipole-bound anion.

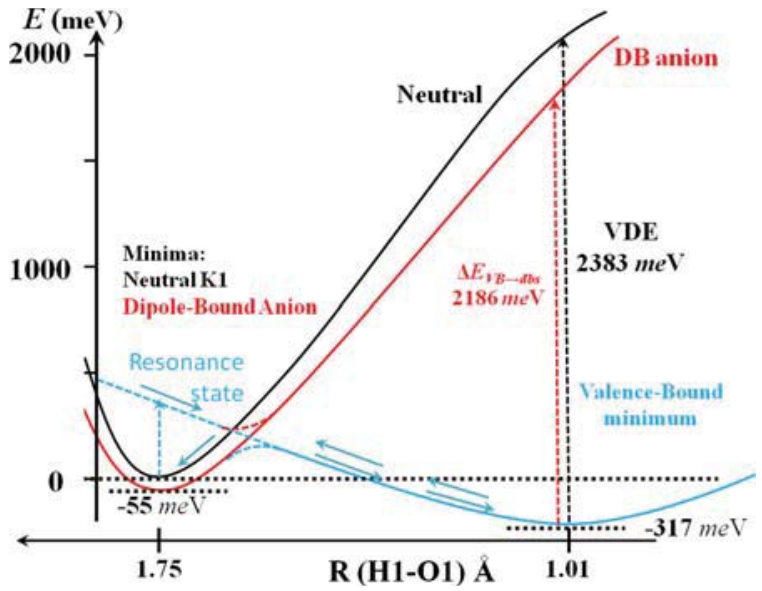

Figure 5. Energy profile depicting the neutral (black), dipole-bound (red), and valence (blue) anionic potential energy surfaces of AA. Energies $(\mathrm{meV})$ computed at the $\mathrm{CCSD}(\mathrm{T}) / \mathrm{ADZ}+\mathrm{DF}$ level. (Left) Dipole-bound anionic minimum and the local minimum of the neutral (K1). (Right) Global minimum of the valence anion.

With $\mathrm{H} 1$ transferred from $\mathrm{O} 2$ to $\mathrm{O} 1$, the valence anion supports a minimum, which is characterized by a VDE of 2383 $\mathrm{meV}$. The valence anion is adiabatically bound with respect to the neutral $\mathrm{K} 1$ by $317 \mathrm{meV}$ (in terms of electronic energies). A vertical electronic excitation energy from the valence- to the dipole-bound anionic state, $\Delta E_{\mathrm{VB} \rightarrow \mathrm{dbs}}$, is $2186 \mathrm{meV}$. In future experiments, one might want to probe the molecular dynamics of $\mathrm{AA}^{-}$upon this electronic excitation.

The singly occupied molecular orbitals in the DB and VB anions, each at its minimum energy structure, are illustrated in Figure 6 . The former orbital is very diffuse and localized on the

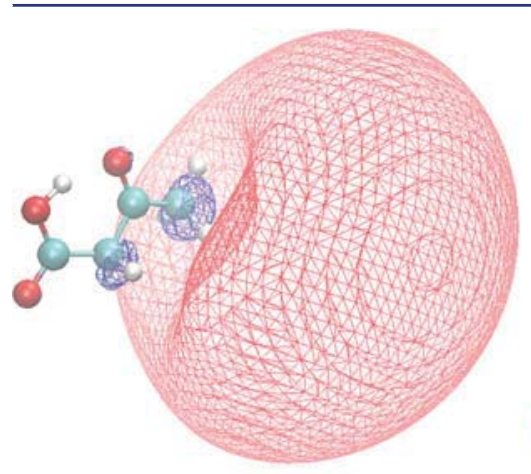

(a) Dipole bound

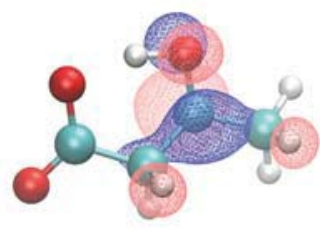

(b) Valence bound
Figure 6. Orbital occupied by an excess electron in the DB and VB anions of AA plotted with a fraction of electron density $\left(F_{\mathrm{e}}\right)$ equal to $0.6^{42}$

positive pole of the molecular dipole, which proves to be a hydrophobic group $\mathrm{CH}_{3}$. The latter is a $\pi$ orbital localized around the $\mathrm{C} 2$ atom, with bonding interactions involving the neighboring carbon atoms and an antibonding interaction between $\mathrm{C} 2$ and $\mathrm{O} 1$. The unpaired electron in the valence anion is localized in the vicinity of $\mathrm{C} 2 \mathrm{O} 1 \mathrm{H}$, but the excess charge is localized on $\mathrm{C} \mathrm{OO}^{-}$.

$G_{M_{\mathrm{dbs}}^{-}}$differs from $G_{M}$ by shortening the $\mathrm{O} 1 \cdots \mathrm{H} 1$ distance by $0.036 \AA$, increasing the $\mathrm{O} 1-\mathrm{H} 1-\mathrm{O} 2$ angle by $1.6^{\circ}$, and an elongation of the $\mathrm{O} 2-\mathrm{H} 1$ distance by $0.003 \AA$, see Figures 2 and 3 . Overall it is a small step toward proton transfer, a nascent zwitterionization. The dipole moment of neutral AA 
increases by $0.16 \mathrm{D}$ upon these geometrical distortions, a typical increase for dipole-bound anionic states.

The vertical electron binding energies for the dipole-bound anionic state, obtained with indirect and direct methods, are reported in Table 2 for the $G_{M}, G_{M_{\mathrm{dbs}}^{-}}$, and the $G_{M_{\mathrm{VB}}^{-}}$geometries.

Table 2. Vertical Electron Binding Energies (meV) of the Dipole-Bound Anionic State at the $G_{M}, G_{M_{\mathrm{dbs}}^{-}}$, and $G_{M_{\mathrm{VB}}^{-}}$ Geometries Using the CCSD/ADZ+DF Optimal Geometries

\begin{tabular}{clccc}
\multirow{2}{*}{ method } & & \multicolumn{3}{c}{$\mathrm{EBE}$} \\
\cline { 3 - 5 } indirect & $\mathrm{SCF}$ & $G_{M}$ & $G_{M_{\mathrm{dbs}}^{-}}$ & $G_{M_{\mathrm{VB}}^{-}}$ \\
& $\mathrm{MP2}$ & 24.06 & 26.89 & 127.68 \\
& $\mathrm{CCSD}$ & 52.81 & 36.31 & 217.72 \\
& $\mathrm{CCSD}(\mathrm{T})$ & 52.34 & 56.59 & 196.85 \\
\multirow{3}{*}{ direct EA } & KT & 21.59 & 56.55 & 197.04 \\
& EPT third & 43.63 & 47.46 & 245.07 \\
direct IP & KT & 26.32 & 29.78 & 135.79 \\
& EPT third & 45.27 & 50.45 & 247.84 \\
\hline
\end{tabular}

Starting from "indirect" electron binding energies, the SCF contribution represents only $46-65 \%$ of the $\operatorname{CCSD}(\mathrm{T})$ results, illustrating the role of electron correlation effects in dipolebound anionic states. The differences between the CCSD and the $\operatorname{CCSD}(\mathrm{T})$ results do not exceed $0.2 \mathrm{meV}$. The electron binding energies obtained in "direct" approaches start from the Koopmans' theorem estimations; thus, they neglect orbital relaxation and electron correlation effects. The differences between the KT and the SCF "indirect" results are small, indicating that orbital relaxation effects are minor for the dipole-bound anionic state. The EPT third-order results are in good agreement with the "indirect" $\operatorname{CCSD}(\mathrm{T})$ results. The role of the second-order dispersion interaction between the loosely bound electron and the electrons of AA is illustrated in Table S4.

Various estimations of adiabatic electron affinity for this anionic state are reported in Table 3. The $\operatorname{CCSD}(\mathrm{T})$ and

Table 3. Adiabatic Electron Affinities (meV) of the DB and VB Anions Calculated at Different Levels of Theory with the CCSD-Optimized Geometries ${ }^{a}$

\begin{tabular}{lrrrrrrrr} 
& \multicolumn{4}{c}{ AEA (electronic) } & & $\Delta E_{\text {vib }}$ & & AEA \\
\cline { 2 - 4 } & \multicolumn{1}{c}{ SCF } & MP2 & CCSD & $\operatorname{CCSD}(\mathrm{T})$ & & $\operatorname{CCSD}$ & & $\operatorname{CCSD}(\mathrm{T})$ \\
DB & 8.60 & 38.88 & 54.15 & 54.56 & & 2.63 & & 57.19 \\
VB & -182.80 & 286.26 & 289.55 & 317.02 & & 17.27 & & 334.29
\end{tabular}

${ }^{a}$ All calculations with the $\mathrm{ADZ}+\mathrm{DF}$ basis set.

CCSD results are very similar, approximately $54 \mathrm{meV}$, and the zero-point vibrational correction further stabilizes the anion by $3 \mathrm{meV}$. The decomposition of the electronic component of AEA into the $\Delta E_{M}\left(G_{M^{-}}\right)$and VDE terms (eq 5, Table 4) illustrates a very small geometrical distortion of the molecular framework upon the excess electron attachment and the dominant role of VDE.

In contrast to the neutral AA, which supports several lowlying minimum energy structures, the valence anion of AA supports one distinct global minimum illustrated on the right side of Figure 3. Other minima (local) are less stable by more than $12 \mathrm{kcal} / \mathrm{mol}$ and are characterized by negative values of AEA. They display VDEs exceeding $2.9 \mathrm{eV}$, thus much higher
Table 4. Electronic component $(\mathrm{CCSD}(\mathrm{T}) / \mathrm{ADZ}+\mathrm{DF})$ of AEA (meV) decomposed into the $\Delta E_{M}\left(G_{M^{-}}\right)$and VDE terms

\begin{tabular}{crrr} 
& $-\Delta E_{M}\left(G_{M^{-}}\right)$ & \multicolumn{1}{c}{$\mathrm{VDE}$} & \multicolumn{1}{c}{$\mathrm{AEA}$} \\
DB & -1.99 & 56.55 & 54.56 \\
VB & -2065.69 & 2382.71 & 317.02 \\
\hline
\end{tabular}

than the VDE of VB, see Table S6 and Figure S4. We also searched for valence anions associated with the enol structures. There were no electronically bound valence anions around the equilibrium structures of E1 and E3. Even upon transferring the $\mathrm{O} 1 \mathrm{H}$ proton to the carboxylic group, the valence anions remained electronically unbound. Thus, from now on, our discussion will be limited to VB illustrated on the right side of Figure 3.

In the VB minimum, the $\mathrm{H} 1$ proton is bound to $\mathrm{O} 1$ and the $\mathrm{O} 1 \mathrm{H} 1 \cdots \mathrm{O} 2$ hydrogen bond is very short, $R(\mathrm{H} 1 \mathrm{O} 2)=1.583 \AA$. The intramolecular hydrogen bond is more linear than in the neutral or dipole-bound anion by $6-8^{\circ}$. When compared with the $\mathrm{K} 1$ neutral, there is a significant elongation of the $\mathrm{C} 2 \mathrm{O} 1$ distance by $0.146 \AA$ and a shortening of $\mathrm{C} 4 \mathrm{O} 2$ by $0.064 \AA$, consistent with a redistribution of double bonds upon tautomerization. Finally, the $\mathrm{CH}_{3}$ group is rotated by ca. $58^{\circ}$ in comparison with $\mathrm{K} \mathbf{1}$.

The VDEs obtained using "indirect" methods and "direct" EPT span a reasonably narrow range of $2300-2600 \mathrm{meV}$ (Table 5). The Koopmans' theorem EA and IP values are 713

Table 5. Values of VDE ( $\mathrm{meV}$ ) for the Valence Anion of AA

\begin{tabular}{clr} 
method & & VDE \\
indirect & SCF & 2400.48 \\
& MP2 & 2347.34 \\
& CCSD & 2482.36 \\
& CCSD $(\mathrm{T})$ & 2382.71 \\
direct EA & KT & 713.43 \\
direct IP & EPT third & 2304.34 \\
& KT & 4200.09 \\
& EPT third & 2602.50 \\
\hline
\end{tabular}

and $4200 \mathrm{meV}$, respectively, while the indirect SCF value is $2400 \mathrm{meV}$, demonstrating that orbital relaxation effects are critically important for this anionic state. Electron correlation effects, on the other hand, do not contribute much to the VDE value. The anion should be readily formed in anion sources, because its adiabatic electron affinity is modest but positive, i.e., $334 \mathrm{meV}$, after inclusion of the zero-point vibrational correction (Table 3). The electronic contribution to AEA can be analyzed in terms of eq 5 (Table 4). Proton transfer from $\mathrm{O} 2$ to $\mathrm{O} 1$ is accompanied by a significant increase of the energy of the neutral $\left(\Delta E_{M}\left(G_{M_{\mathrm{VB}}^{-}}\right)=2066 \mathrm{meV}\right)$. This energy increase is, however, outweighed by the VDE of $2383 \mathrm{meV}$. The modest electronic contribution to AEA of $317 \mathrm{meV}$ results from a cancellation of the $\Delta E_{M}\left(G_{M_{\mathrm{VB}}^{-}}\right)$and VDE terms.

\section{EXPERIMENTAL RESULTS}

5.1. Photoelectron Spectroscopy Results for $\mathrm{AA}^{-}$and $(\mathrm{AA})_{2}{ }^{-}$. Parent anions of AA were prepared using two rather different anion source environments. Figure 7 presents a representative mass spectrum of the anions produced by our pulsed, laser photoemission source. In addition to $\mathrm{AA}^{-}$, the spectrum shows $\left(\mathrm{AA}-\mathrm{H}_{2} \mathrm{O}\right)^{-}$. Our preliminary computational results for several isomers of $\left(\mathrm{AA}-\mathrm{H}_{2} \mathrm{O}\right)^{-}$indicate that some of 


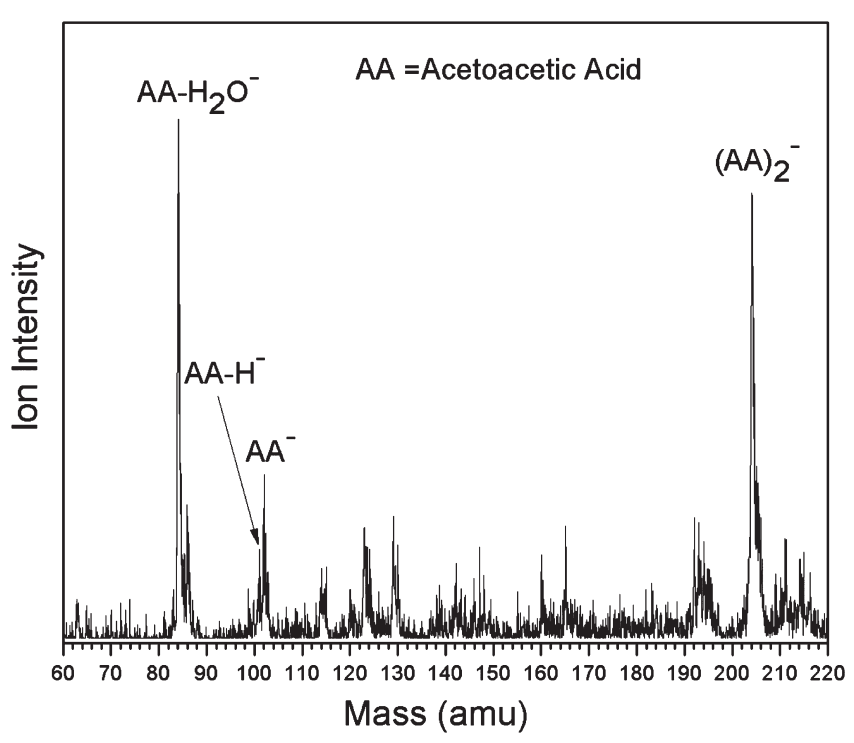

Figure 7. Mass spectrum of anions observed in these experiments.

them are adiabatically bound with the VDEs exceeding $1.4 \mathrm{eV}$. Our complete results for the anions of $\mathrm{AA}-\mathrm{H}_{2} \mathrm{O}$ will be presented in a future report. The mass spectrum also shows $(\mathrm{AA})_{2}{ }^{-}$.

The photoelectron spectrum of the $\mathrm{AA}^{-}$parent anion measured on our continuous photoelectron spectrometer and recorded with $2.54 \mathrm{eV}$ photons is presented in Figure 8. This

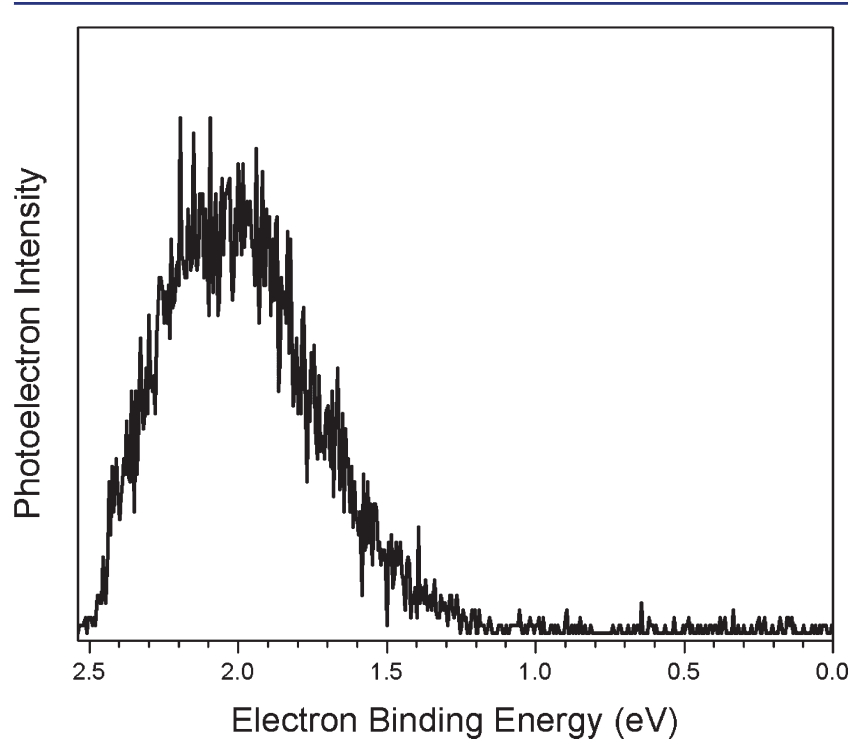

Figure 8. Photoelectron spectrum of the $\mathrm{AA}^{-}$parent anion recorded with $2.54 \mathrm{eV}$ photons on our continuous anion photoelectron spectrometer.

spectrum consists of a broad, featureless band with an onset at $\mathrm{EBE} \approx 1.2 \mathrm{eV}$ and an intensity maximum at $\mathrm{EBE} 2.04 \mathrm{eV}$. This latter quantity corresponds to the VDE of $\mathrm{AA}^{-}$. The calculated adiabatic electron affinity of $0.33 \mathrm{eV}$ is much smaller than the onset, suggesting that the $0-0$ transition has a diminishingly small intensity due to poor Franck-Condon overlap between the $\mathrm{AA}^{-}$anion and its neutral counterpart. The calculated VDE of $2.38 \mathrm{eV}$ is larger by $0.34 \mathrm{eV}$ than the measured intensity maximum. The source of this discrepancy is discussed below.
The photoelectron spectrum of the $\mathrm{AA}^{-}$parent anion measured on the pulsed photoelectron spectrometer and recorded with $3.49 \mathrm{eV}$ photons is presented in Figure 9. This

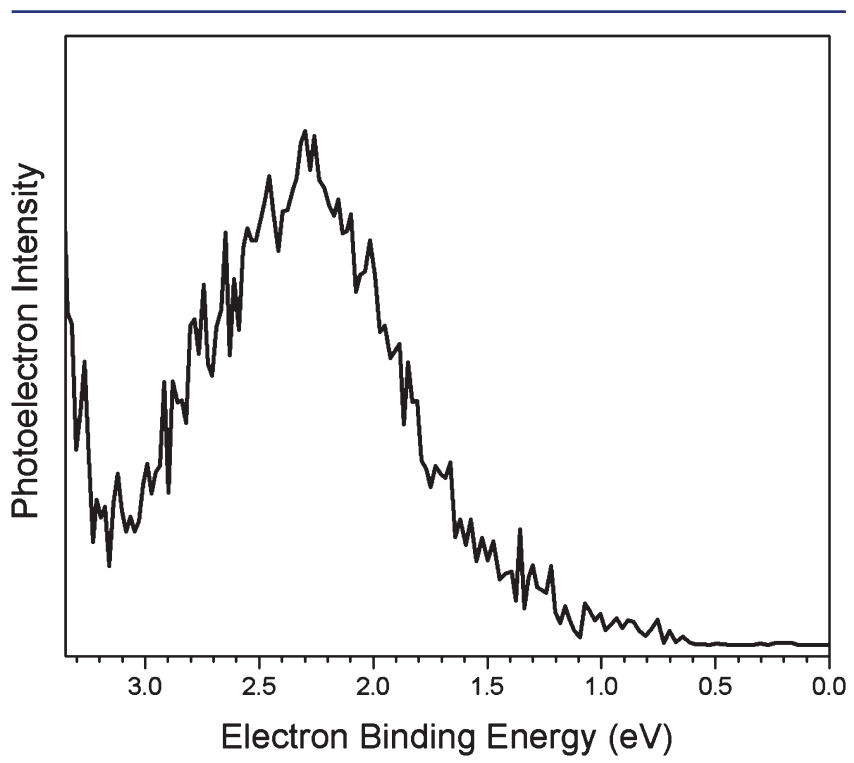

Figure 9. Photoelectron spectrum of the $\mathrm{AA}^{-}$parent anion recorded with $3.49 \mathrm{eV}$ photons on the pulsed photoelectron apparatus.

spectrum also consists of a broad, featureless band with an onset at $\mathrm{EBE} \approx 1.2 \mathrm{eV}$ but with an intensity maximum at $\mathrm{EBE}$ $2.30 \mathrm{eV}$. This latter quantity again corresponds to the VDE of $\mathrm{AA}^{-}$. This value of $\mathrm{VDE}$ is in good agreement with the calculated value of $2.38 \mathrm{eV}$. This spectrum does not display a pronounced intensity increase in the $2.9-3.0 \mathrm{eV}$ range, where the electron vertical detachment energies for the valence anions other than VB are predicted to appear (see Figure S4).

For the most part, the two photoelectron spectra are the same, indicating that both source environments produced the same parent $\mathrm{AA}^{-}$species. The main difference lies in the slightly different VDE values obtained on different apparatuses. The difference between the two measured VDE values is attributed to the electron transmission function roll-off inherent for low kinetic energy (high EBE) electrons. This effect comes into play more strongly for a lower versus a higher photon energy, since a lower photon energy puts more of the spectrum in the low EKE region of the spectrum. On the continuous apparatus, whose photon energy was $2.54 \mathrm{eV} /$ photon, this had the effect of attenuating the still rising photoelectron band, making its intensity maximum appear to occur at a slightly lower EBE value. By contrast, the pulsed apparatus, which utilized a photon energy of $3.49 \mathrm{eV} /$ photon, was not significantly affected in this way. Thus, we believe that the more reliable value for the $\mathrm{VDE}$ of $\mathrm{AA}^{-}$is $2.3 \mathrm{eV}$, close to that predicted by theory.

We also measured the photoelectron spectrum of the parent dimer anion, $(\mathrm{AA})_{2}^{-}$, and it is presented in Figure 10. This spectrum also exhibits a single broad band, and it has a similar width to that of the monomeric $\mathrm{AA}^{-}$. Interestingly, its VDE value, at $1.7 \mathrm{eV}$, is considerably lower than that of the monomer, indicating that the additional AA molecule is not simply solvating $\mathrm{AA}^{-}$, i.e., it is not a simple anion-molecule complex.

5.2. Electron Energy-Loss Spectroscopy Results for AA. Our electron energy-loss spectrum for AA is shown in 


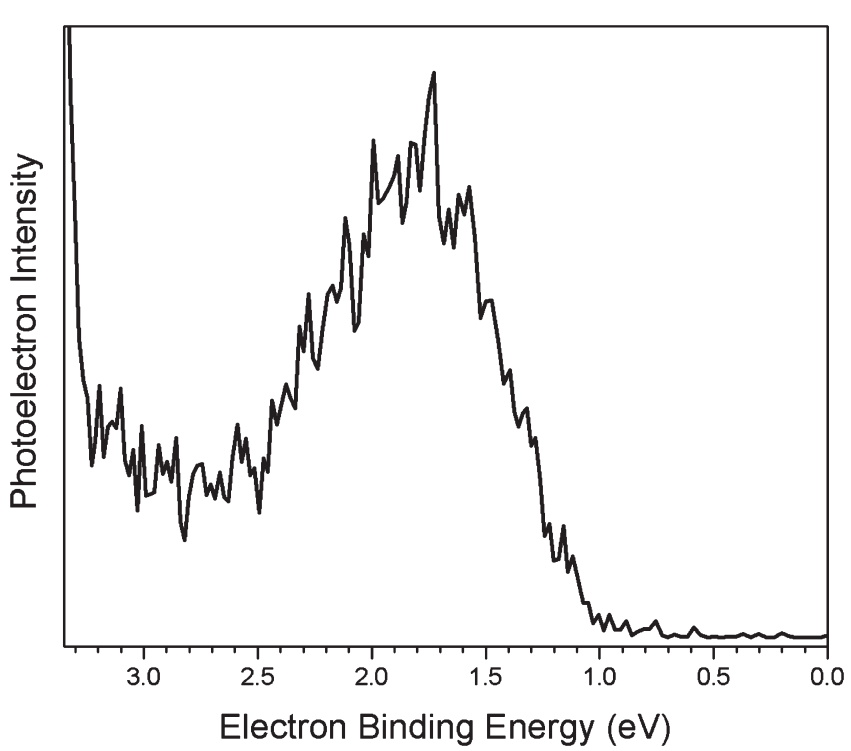

Figure 10. Photoelectron spectrum of $(\mathrm{AA})_{2}{ }^{-}$parent anion recorded with $3.49 \mathrm{eV}$ photons on the pulsed photoelectron apparatus.

Figure 11. The hypothetical processes induced by the attachment of an electron onto neutral K1 are suitably

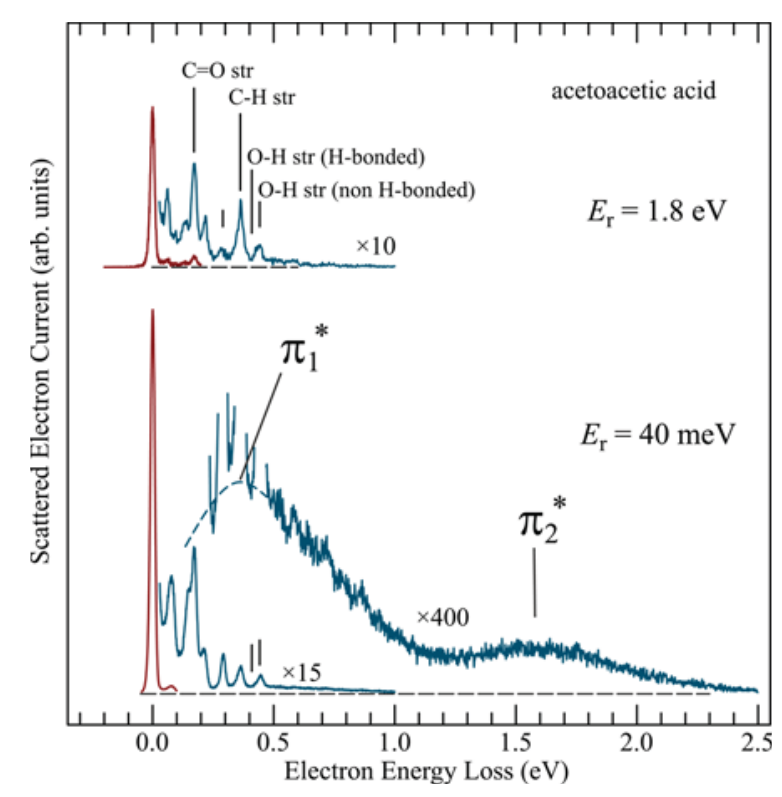

Figure 11. Spectra showing the yields of electrons with the specified residual energies $E_{\mathrm{r}}$ plotted as a function of energy loss.

visualized by the diagram in Figure 5. Vertical electron attachment, indicated by the vertical blue dashed arrow, transfers the nuclear wave packet to the repulsive part of the valence anion potential surface-a resonance because it is subject to autodetachment. The present discussion concentrates on the lowest shape (i.e., not core excited) resonance with temporary occupation of the $\pi_{1}^{*}$ orbital. The nuclear wave packet then starts to relax by sliding down the repulsive surface, in competition with rapid autodetachment. Detachment of an electron at an early stage of the relaxation leads to a vibrationally excited final state of the neutral molecule. Since the detachment rate during the initial relaxation of the nuclei is faster than the nuclear motion (this is indicated by the absence of boomerang structure in the spectra), ${ }^{52}$ this process leads to (i) excitation of primarily low quanta of the vibration and (ii) pronounced selectivity with respect to the modes, with those modes being primarily excited along which the anion potential has a large slope at the point of attachment. This process has been termed "specific" vibrational excitation ${ }^{53}$ and gives rise to the sharp vibrational peaks on the left side of the spectra in Figure 11. A fraction of the nuclear wave packet survives until it reaches portions of the potential surface where the detachment is slow or the ion is even bound, allowing intramolecular vibrational redistribution (IVR) which "wipes out" the initial mode specificity, leading to the excitation of a quasi-continuum of high-lying vibrational levels, accompanied by detachment of a very slow electron. This process has been termed "unspecific" vibrational excitation ${ }^{53}$ and gives rise to the structureless humps in the lower spectrum in Figure 11. One could also term the detachment in the initial phases of the relaxation "nearly vertical" and in the later phases, after IVR, "nearly horizontal". The latter process gives rise to the capacity of the resonances to quasi-thermalize the incident electrons. The "unspecific" vibrational excitation and the corresponding humps in the spectra showing the yield of very slow electrons at incident electron energies corresponding to resonances are found in all molecules larger than diatomic. ${ }^{33}$ The effect is usually weak but becomes more pronounced for large molecules. The two humps in the lower spectrum in Figure 11 indicate a $\pi_{1}^{*}$ (LUMO) resonance at $0.4 \mathrm{eV}$ and a $\pi_{2}^{*}(\mathrm{LUMO}+1)$ resonance at $1.6 \mathrm{eV}$.

An interesting effect was observed in the formic acid dimer where the "unspecific" vibrational excitation band in the yield of quasi-thermal electrons was anomalously large, 20 times larger than in the monomer. ${ }^{18}$ It was interpreted as a manifestation of a very fast intermolecular proton (or hydrogen) transfer in the resonance, which gave a competitive edge to relaxation over electron detachment, thus increasing the yield of the unspecific process.

It may be noted as a side remark that stretching of the $\mathrm{O}-\mathrm{H}$ bond in the formic acid monomer yields $\mathrm{HCOO}^{-}+\mathrm{H}$ (dissociative electron attachment, DEA), the mechanism of which we studied recently. ${ }^{54}$ The intermolecular (in the case of the formic acid dimer) and intramolecular (in the case of AA) proton transfer may be viewed as a "frustrated" DEA.

The question posed here is whether an anomalously high intensity of the "unspecific" vibrational excitation hump, linked to intramolecular proton transfer, is also observed in AA. The spectra in Figure 11 show that a hump is indeed observed, but to decide whether it may be called anomalously high requires at least an approximate quantitative consideration. For this purpose we determined the ratio of the signals integrated under the discrete narrow vibrational peaks (representative for the weakly relaxed specific process) and the structureless hump (representative for the fully relaxed unspecific process).

To bypass a problem given by the overlap of the unspecific and the specific energy ranges below $0.5 \mathrm{eV}$, the unspecific signal is extrapolated visually to low energies as indicated by the dashed curve in Figure 11. The shape of the extrapolated section is guided by the depths of the valleys between the specific vibrational peaks down to $0.25 \mathrm{eV}$ and by the expected shape of a Franck-Condon band below that. The integral under the smooth band, including the extrapolated section, is taken as the unspecific contribution. The specific contribution is the integral under the total measured signal minus the unspecific contribution. Both integrals were taken between 
0.035 and $1.2 \mathrm{eV}$. The result is that the integral under the structureless band is about 3 times less than the integral under the narrow structures. This can be compared to the formic acid dimer where the integral under the structureless band is about 3 times larger than the integral under the narrow structures. The intensity of the unspecific signal relative to the specific signal is thus 9 times smaller in AA when compared to formic acid dimer. The unspecific signal in AA is thus not anomalously high and cannot be taken as evidence for intramolecular proton transfer. The unspecific/specific ratio in AA is comparable, only marginally 2 times larger than that in the formic acid monomer (without intramolecular proton transfer). This would be expected for the "normal" (i.e., given by IVR and not by fast intramolecular transfer) unspecific signal which increases with increasing size of the molecule.

The capacity of the $\pi_{1}^{*}$ resonance in AA to quasi-thermalize electrons is thus not large enough to provide positive evidence for an intramolecular proton transfer fast enough to compete with autodetachment. The anion photoelectron spectra and the calculations show beyond doubt that the equilibrium structure of the valence anion is proton transferred, however. The combined PES and EELS experimental evidence thus indicates that the electron-induced transfer occurs but slower than in formic acid dimer, so that it does not efficiently compete with the fast autodetachment. The reason may be that only a fraction of the target $\mathrm{AA}$ is in the $\mathrm{H}$-bonded conformation suitable for fast proton transfer.

This is indicated by the computational results detailed in Table $\mathbf{1}$. The $\mathbf{K} \mathbf{1}$ conformer, which is capable to relay $\mathrm{H} 1$, is thermodynamically disfavored. This conformer is also kinetically difficult to access from the more populated $\mathrm{K} 3$ and $\mathrm{K} 5$ conformers due to a high-barrier TS1 (see Figure 4). Thus, a rotation of the $\mathrm{O} 2 \mathrm{H} 1$ bond to make a H-bonded keto conformer is expected to be slow in comparison with electron autodetachment. In consequence, the temporary anion typically autodetaches before finding a refuge on the right side of Figure 5 , where the anionic state is bound. Another neutral AA with a bridging hydrogen, E1, is expected to be more common than K1 (Table 1), but it does not support an electronically bound valence anion either with or without a proton transferred from $\mathrm{O} 1 \mathrm{H}$ to $\mathrm{O} 3$. Thus, the slope of the anion potential on the left side of Figure 5 is expected to be smaller than for $\mathbf{K} \mathbf{1}$, and there is no refuge where the anion would be electronically bound.

The above interpretation is consistent with the discrete vibrational energy-loss spectra on the left side of both traces in Figure 11, where nearly no intensity is observed for H-bonded $\mathrm{O}-\mathrm{H}$ stretch. Our computational results confirm that the $\mathrm{O}-$ Hs engaged in hydrogen bonds have lower frequencies (3433 (K1) and $3304 \mathrm{~cm}^{-1}$ (E1)) than those with free O-Hs (3731 (K3), 3736 (K5), and $3740 \mathrm{~cm}^{-1}$ (E1)). The low-frequency $\mathrm{O}-\mathrm{H}$ stretches are not visible in the spectrum because $\mathbf{K} \mathbf{1}$ and E1 are minor constituents of the gas phase AA. Additionally, even if $\mathrm{E} 1$ was present, its $\mathrm{H}$-bonded $\mathrm{O}-\mathrm{H}$ stretch would not be pronounced because the slope of the anion potential along the proton transfer coordinate is low at the point of electron attachment.

\section{DISCUSSION}

The computational results indicate that the global minimum of the acetoacetic acid valence anion corresponds to an ol structure, with a proton transferred from the carboxylic to the keto group. We conclude that binding an excess electron on the $\pi_{1}^{*}$ valence orbital changes the localization of the proton in acetoacetic acid, provided the anion becomes fully relaxed. The valence anion is characterized by a significant electron vertical detachment energy of $2383 \mathrm{meV}$ but only a modest adiabatic electron affinity of $334 \mathrm{meV}$.

Parent anions of acetoacetic acid were successfully prepared using two different source environments. The photoelectron spectra consist of a broad, featureless band with an onset at an electron binding energy of ca. $1.2 \mathrm{eV}$. The intensity maximum recorded with $3.49 \mathrm{eV}$ photons is at $2.30 \mathrm{eV}$, in good agreement with the computed VDE of $2.38 \mathrm{eV}$ for the fully relaxed anion. The calculated adiabatic electron affinity of $0.33 \mathrm{eV}$ is much smaller than the onset of the spectra, indicating that the $0-0$ transition has a very small intensity due to poor FranckCondon overlap.

The electron energy-loss spectra do show a broad structureless band in the yield of quasi-thermalized electrons $(40 \mathrm{meV})$ following an electron attachment into the $\pi_{1}^{*}$ resonance. This band indicates a rapid IVR process in the resonance, but in contrast to the formic acid dimer case the band is not anomalously strong and thus does not provide evidence of the IVR process being promoted by an ultrafast proton transfer in the resonance. We explain it as a consequence of the fact that only the $\mathbf{K} \mathbf{1}$ conformer is prearranged to relay a proton across the $\mathrm{H}$-bonded bridge. However, contrary to naive chemical intuition, this conformer is present in only a small fraction in the target gas of the energy-loss experiment. Evidence for the low population comes from the calculations and from the near absence of the $\mathrm{H}$ bonded $\mathrm{O}-\mathrm{H}$ stretch in the energy-loss spectra. The global minimum for the neutral formic acid dimer, on the other hand, is supported by a cyclic hydrogen bond, and thus, it is prearranged to transfer a proton upon an excess electron attachment. $^{18,19}$

The agreement between the measured $(2.30 \mathrm{eV})$ and the calculated $(2.38 \mathrm{eV})$ values of VDE indicates that $\mathrm{AA}^{-}$formed in the microplasma sources of the PES experiments is the fully relaxed valence-bound anion $\mathrm{VB}$, with the $-\mathrm{COOH}$ proton transferred to the keto group. In these experiments the vapor of AA is expanded with a noble gas into vacuum and electrons are injected in the high-pressure portion of the jet. Three-body collisions (noble gas) are needed to cool hot, short-lived autodetaching species. A typical flight time to the mass selector and to the photodetachment region is $5-20 \mu \mathrm{s}$, depending on the mass of the anion and on the energy at which it was accelerated. Thus, the temporary species have roughly a $10 \mu \mathrm{s}$ temporal window to undergo ion-molecule reactions, form larger clusters, and cool down. The occurrence of complex chemical transformations has indeed been demonstrated in our past studies on anions of nucleic acid bases. ${ }^{55}$ The most stable valence anions are formed upon proton transfer from nitrogen to carbon atoms. The barriers for unimolecular transformations were found to be prohibitively high, and dissociative electron attachment followed by ion-molecule reactions were invoked to justify formation of these unusual species.

Formation of $\mathrm{AA}^{-}$in the microplasma source does not rely on the presence of $\mathrm{K} \mathbf{1}$ in the expanding mixture of $\mathrm{AA}$ and the noble gas. The formation mechanism might be more complex than a simple electron attachment to AA followed by a rapid proton transfer. There is a temporal window of several microseconds for the anionic species to rotate the $\mathrm{O} 2 \mathrm{H} 1$ bond and reach the $\mathrm{H}$-bonded bridge structure, transfer the proton, cool down in the course of collisions with noble gas 
atoms, and relax into the lowest vibrational state of the global minimum of VB.

In contrast, the EELS experiments probe a distribution of neutral molecules in the gas phase. The H-bonded $\mathbf{K} \mathbf{1}$ conformer is minor in the liquid phase, ${ }^{26}$ and accessing it in the gas phase is obstructed by a transition state of $9.2 \mathrm{kcal} / \mathrm{mol}$. Short-lived autodetaching anionic species have a temporal window comparable with the resonance lifetime, thus femtoseconds, to transfer a proton in unimolecular processes. A rotation of the $\mathrm{O} 2 \mathrm{H} 1$ bond is too slow to reach the $\mathrm{H}$-bonded bridge structure suited for relaying $\mathrm{H} 1$ to the keto group and sheltering the anion in the electronically bound region of the potential energy surface. As a result, the temporary anion of AA decays primarily through a "nearly vertical" autodetachment.

\section{SUMMARY}

We searched for the most stable tautomers and conformers of the neutral and anionic acetoacetic acid using a potential energy surface scanning tool (PESST). ${ }^{27}$ The neutral AA supports minima for the keto and enol tautomers. Our $\operatorname{CCSD}(\mathrm{T})$ electronic energies corrected for zero-point vibrations and thermal contributions to the Gibbs free energy indicate that the keto conformers without the intramolecular hydrogen bond are more stable than the conformers with the intramolecular hydrogen bond (keto or enol). The spread of Gibbs free energies is, however, very narrow, less than $1 \mathrm{kcal} / \mathrm{mol}$ at standard conditions.

We paid particular attention to a keto conformer K1 with the intramolecular hydrogen bond and thus susceptible to intramolecular carboxylic-to-keto proton transfer. It belongs to a grouping of the most stable conformers of the neutral AA and proved to be the most polar, with a dipole moment of 5.4 D. K1 is separated from other keto conformers by a relatively high barrier exceeding $9 \mathrm{kcal} / \mathrm{mol}$. The barriers separating other conformers are much smaller and do not exceed $1.1 \mathrm{kcal} / \mathrm{mol}$. K1 supports a dipole-bound anion with an electron vertical detachment energy of $57 \mathrm{meV}$. The dipole-bound anion remains bound upon the intramolecular carboxylic-to-keto proton transfer.

The valence anion of acetoacetic acid undergoes proton transfer upon an excess electron attachment. The distinct global minimum is supported by a short $(\mathrm{H} \cdots \mathrm{O}$ distance of $1.58 \AA$ ) intramolecular hydrogen bond between the hydroxy and carboxylate groups. The valence anion is not only vertically $(2383 \mathrm{meV})$ but also adiabatically $(334 \mathrm{meV})$ bound. The electronic excitation energy from the valence- to dipole-bound state is $2186 \mathrm{meV}$. Dynamics of the anion excited to the repulsive wall of the dipole-bound state can be explored in future experiments.

The photoelectron spectra from the continuous and pulsed photoelectron spectrometers were obtained with 2.54 and 3.49 $\mathrm{eV}$ photons, respectively. The spectrum obtained with $3.49 \mathrm{eV}$ photons from the pulsed apparatus is more reliable and displays a broad, featureless band with an onset at an electron binding energy of ca. $1.2 \mathrm{eV}$ and an intensity maximum at $2.30 \mathrm{eV}$. The reported spectra do not provide evidence for the dipole-bound anion. The photoelectron spectrum of the parent dimer anion of acetoacetic acid shows a vertical detachment energy at 1.7 $\mathrm{eV}$, which is considerably lower than that of the monomer, indicating that the additional monomer is not simply solvating the monomeric anion.

The electron energy-loss spectrum of acetoacetic acid displays narrow vibrational peaks representative of "nearly vertical" electron detachments and structureless humps representative of "nearly horizontal" detachments. The ratio of signals integrated under the discrete vibrational peaks and the structureless hump do not provide evidence for an intramolecular proton transfer fast enough to compete with autodetachment. The reason is that the conformer capable to relay a proton, i.e., $\mathbf{H}$-bonded $\mathbf{K} \mathbf{1}$, is thermodynamically disfavored and kinetically difficult to access from the more populated but non-H-bonded keto conformers. This is confirmed by the near absence of the $\mathrm{H}$-bonded $\mathrm{O}-\mathrm{H}$ stretch in the energy-loss spectra.

Our results expose the importance of time scale and local environment in electron-driven proton transfer. The microplasma sources of anions in the PES experiments offer a temporal window of several microseconds to reach a H-bonded bridge structure, transfer the proton, cool down the temporary anion in course of collisions with noble gas atoms, and settle in the lowest vibrational state of the anionic global minimum. EELS experiments, on the other hand, probe a distribution of neutral molecules in the gas phase. Short-lived autodetaching anionic species have a temporal window comparable with the resonance lifetime, thus femtoseconds, to transfer a proton in unimolecular processes. This takes place in the formic acid dimer, because its global minimum is supported by a cyclic hydrogen bond and thus prearranged to relay a proton. ${ }^{18,19} \mathrm{~A}$ $\mathrm{H}$-bonded bridge is not available in the most stable conformers of neutral acetoacetic acid, and the temporary anion decays primarily through a "nearly vertical" autodetachment.

Before closing we comment on the electron-driven proton transfer scheme in acetoacetic acid illustrated in the bottom of Figure 1. The right-hand side of it is true; the fully relaxed valence anion has an ol structure, is adiabatically bound, and displays a significant vertical detachment energy of $2.3 \mathrm{eV}$. The left and central parts are problematic. The neutral K1 would indeed facilitate proton transfer due to the intramolecular $\mathrm{H}$ bond bridge. Unfortunately, this conformer is not dominant in the gas phase at standard conditions. The spread of Gibbs free energies of the keto and enol tautomers and their conformers is very narrow, less than $1 \mathrm{kcal} / \mathrm{mol}$ at standard conditions, and the determination of major constituents of the gas-phase AA would be challenging without a combinatorial/computational tool PESST. ${ }^{27}$

\section{ASSOCIATED CONTENT}

\section{S Supporting Information}

The Supporting Information is available free of charge on the ACS Publications website at DOI: 10.1021/jacs.5b08134.

Construction of the linear synchronous path connecting $G_{M_{\mathrm{dbs}}^{-}}$with $G_{M_{\mathrm{VB}}^{-}}$. The absolute electronic energies and zero-point vibration energy of the $\mathbf{K} \mathbf{1}$ conformer; relative energies of keto conformers and transition states separating them; dipole moments of keto conformers; relative energies of enol conformers; incremental electronic binding energies of the dipole-bound state at various geometries; contribution from the Amber force field to the energies of $\mathbf{K 1}, \mathbf{K} 3$, and $\mathbf{K 5}$; energetics of valence-bound anions (the absolute electronic energy and zero-point vibration energy of $\mathbf{V B}$, relative electronic energies of other conformers, the VDEs and AEAs); Cartesian coordinates of the most relevant neutral and anionic species (PDF) 


\section{AUTHOR INFORMATION}

\section{Corresponding Authors}

*keolopilezg@mopipi.ub.bw

*M.Gutowski@hw.ac.uk

*angelab@jhu.edu

*ecolli11@jhu.edu

*xzhang54@jhu.edu

*jerb3@jhu.edu

*lectka@jhu.edu

*kbowen@jhu.edu

*Michael.Allan@unifr.ch

\section{Notes}

The authors declare no competing financial interest.

\section{ACKNOWLEDGMENTS}

This work was conducted within the framework of the COST Action CM1301 (CELINA). Z.G.K. was supported by the fellowship from the University of Botswana (UB). This research used resources of the National Energy Research Scientific Computing Center, which is supported by the Office of Science of the U.S. Department of Energy under Contract No. DE-AC02-05CH11231. This material is based (in part) on experimental work supported by the (US) National Science Foundation under Grant No. CHE-1360692 (KHB). This research is a part of project no. 200020-144367/1 of the Swiss National Science Foundation.

\section{REFERENCES}

(1) Chang, C. J.; Chang, M. C.; Damrauer, N. H.; Nocera, D. G. Biochim. Biophys. Acta, Bioenerg. 2004, 1655, 13-28. Special issue dedicated to Jerry Babcock

(2) Sanche, L. Eur. Phys. J. D 2005, 35, 367-390.

(3) Kumar, A.; Sevilla, M. D. Chem. Rev. 2010, 110, 7002-7023.

(4) Dempsey, J. L.; Winkler, J. R.; Gray, H. B. Chem. Rev. 2010, 110, 7024-7039.

(5) Petek, H.; Zhao, J. Chem. Rev. 2010, 110, 7082-7099.

(6) Utke, I.; Hoffmann, P.; Melngailis, J. J. Vac. Sci. Technol. B 2008 , 26, 1197-1276.

(7) Hammes-Schiffer, S. Chem. Rev. 2010, 110, 6937-6938.

(8) Xu, S.; Zheng, W.; Radisic, D.; Bowen, K. H. J. Chem. Phys. 2005, 122, 091103.

(9) Meijer, G.; de Vries, M. S.; Hunziker, H. E.; Wendt, H. R. J. Chem. Phys. 1990, 92, 7625-7635.

(10) Hunig, I.; Plützer, C.; Seefeld, K. A.; Löwenich, D.; Nispel, M.; Kleinermanns, K. ChemPhysChem 2004, 5, 1257-1257.

(11) Bald, I.; Dabkowska, I.; Illenberger, E. Angew. Chem., Int. Ed. 2008, 47, 8518-8520.

(12) Bachorz, R. A.; Harańczyk, M.; Dabkowska, I.; Rak, J.; Gutowski, M. J. Chem. Phys. 2005, 122, 204304.

(13) Gutowski, M.; Dabkowska, I.; Rak, J.; Xu, S.; Nilles, J.; Radisic, D.; Bowen, K. H., Jr. Eur. Phys. J. D 2002, 20, 431-439.

(14) Radisic, D.; Bowen, K. H.; Dabkowska, I.; Storoniak, P.; Rak, J.; Gutowski, M. J. Am. Chem. Soc. 2005, 127, 6443-6450.

(15) Harańczyk, M.; Rak, J.; Gutowski, M.; Radisic, D.; Stokes, S. T.; Bowen, K. H. J. Phys. Chem. B 2005, 109, 13383-13391.

(16) Eustis, S. N.; Radisic, D.; Bowen, K. H.; Bachorz, R. A.; Harańczyk, M.; Schenter, G. K.; Gutowski, M. Science 2008, 319, 936939.

(17) Eustis, S. N.; Whiteside, A.; Wang, D.; Gutowski, M.; Bowen, K. H. J. Phys. Chem. A 2010, 114, 1357-1363.

(18) Allan, M. Phys. Rev. Lett. 2007, 98, 123201.

(19) Gerardi, H. K.; DeBlase, A. F.; Leavitt, C. M.; Su, X.; Jordan, K. D.; McCoy, A. B.; Johnson, M. A. J. Chem. Phys. 2012, 136, 134318.

(20) Stokes, S. T.; Grubisic, A.; Li, X.; Ko, Y. J.; Bowen, K. H. J. Chem. Phys. 2008, 128, 044314.
(21) Kobyłecka, M.; Gu, J.; Rak, J.; Leszczynski, J. J. Chem. Phys. 2008, 128, 044315.

(22) Hay, R. W.; Bond, M. A. Aust. J. Chem. 1967, 20, 1823-1828.

(23) Huang, C.-L.; Wu, C.-C.; Lien, M.-H. J. Phys. Chem. A 1997, 101, 7867-7873.

(24) Pedersen, K. J. J. Am. Chem. Soc. 1929, 51, 2098-2107.

(25) Hoz, S.; Kresge, A. J. J. Phys. Org. Chem. 1997, 10, 182-186.

(26) Grande, K. D.; Rosenfeld, S. M. J. Org. Chem. 1980, 45, 16261628.

(27) Keolopile, Z. G.; Gutowski, M.; Haranczyk, M. J. Chem. Theory Comput. 2013, 9, 4374-4381.

(28) Dunning, T. H. J. Chem. Phys. 1989, 90, 1007-1023.

(29) Kendall, R. A.; Dunning, T. H.; Harrison, R. J. J. Chem. Phys. 1992, 96, 6796-6806.

(30) Gutowski, M.; Simons, J. J. Chem. Phys. 1990, 93, 3874-3880.

(31) Gutowski, M.; Jordan, K. D.; Skurski, P. J. Phys. Chem. A 1998, 102, 2624-2633.

(32) Skurski, P.; Gutowski, M.; Simons, J. Int. J. Quantum Chem. 2000, 80, 1024-1038.

(33) Becke, A. D. J. Chem. Phys. 1993, 98, 5648-5652.

(34) Lee, C.; Yang, W.; Parr, R. G. Phys. Rev. B: Condens. Matter Mater. Phys. 1988, 37, 785-789.

(35) Vosko, S. H.; Wilk, L.; Nusair, M. Can. J. Phys. 1980, 58, 12001211.

(36) Stephens, P. J.; Devlin, F. J.; Chabalowski, C. F.; Frisch, M. J. J. Phys. Chem. 1994, 98, 11623-11627.

(37) Purvis, G. D., III; Bartlett, R. J. J. Chem. Phys. 1982, 76, 19101918.

(38) Bartlett, R. J.; Musial, M. Rev. Mod. Phys. 2007, 79, 291-352.

(39) von Niessen, W.; Schirmer, J.; Cederbaum, L. Comput. Phys. Rep. 1984, $1,57-125$.

(40) Frisch, M. J.; Trucks, G. W.; Schlegel, H. B.; Scuseria, G. E.; Robb, M. A.; Cheeseman, J. R.; Scalmani, G.; Barone, V.; Mennucci, B.; Petersson, G. A.; Nakatsuji, H.; Caricato, M.; Li, X.; Hratchian, H. P.; Izmaylov, A. F.; Bloino, J.; Zheng, G.; Sonnenberg, J. L.; Hada, M.; Ehara, M.; Toyota, K.; Fukuda, R.; Hasegawa, J.; Ishida, M.; Nakajima, T.; Honda, Y.; Kitao, O.; Nakai, H.; Vreven, T.; Montgomery, J. A.Jr.; Peralta, J. E.; Ogliaro, F.; Bearpark, M.; Heyd, J. J.; Brothers, E.; Kudin, K. N.; Staroverov, V. N.; Kobayashi, R.; Normand, J.; Raghavachari, K.; Rendell, A. R.; Burant, J. C.; Iyengar, S. S.; Tomasi, J.; Cossi, M.; Rega, N.; Millam, J. M.; Klene, M.; Knox, J. E.; Cross, J. B.; Bakken, V.; Adamo, C.; Jaramillo, J.; Gomperts, R.; Stratmann, R. E.; Yazyev, O.; Austin, A. J.; Cammi, R.; Pomelli, C.; Ochterski, J. W.; Martin, R. L.; Morokuma, K.; Zakrzewski, V. G.; Voth, G. A.; Salvador, P.; Dannenberg, J. J.; Dapprich, S.; Daniels, A. D.; Farkas, O.; Foresman, J. B.; Ortiz, J. V.; Cioslowski, J.; Fox, D. J. Gaussian 09, Revision D.01; Gaussian Inc.: Wallingford, CT, 2009.

(41) Humphrey, W.; Dalke, A.; Schulten, K. J. Mol. Graphics 1996, $14,33-38$.

(42) Haranczyk, M.; Gutowski, M. J. Chem. Theory Comput. 2008, 4, 689-693.

(43) Dennington, R., Keith, T., Millam, J. GaussView, Version 5; Semichem Inc.: Shawnee Mission, KS, 2009.

(44) Krueger, R. C. J. Am. Chem. Soc. 1952, 74, 5536-5536.

(45) Coe, J. V.; Snodgrass, J. T.; Freidhoff, C. B.; McHugh, K. M.; Bowen, K. H. J. Chem. Phys. 1986, 84, 618-625.

(46) Gerhards, M.; Thomas, O. C.; Nilles, J. M.; Zheng, W.-J.; Bowen, J. K. H. J. Chem. Phys. 2002, 116, 10247-10252.

(47) Neumark, D. M.; Lykke, K. R.; Andersen, T.; Lineberger, W. C. Phys. Rev. A: At., Mol., Opt. Phys. 1985, 32, 1890-1892.

(48) Ho, J.; Ervin, K. M.; Lineberger, W. C. J. Chem. Phys. 1990, 93, 6987-7002.

(49) Rak, J.; Skurski, P.; Simons, J.; Gutowski, M. J. Am. Chem. Soc. 2001, 123, 11695-11707.

(50) Keolopile, Z. G.; Ryder, M. R.; Gutowski, M. J. Phys. Chem. A 2014, 118, 7385-7391.

(51) Cornell, W. D.; Cieplak, P.; Bayly, C. I.; Gould, I. R.; Merz, K. M.; Ferguson, D. M.; Spellmeyer, D. C.; Fox, T.; Caldwell, J. W.; Kollman, P. A. J. Am. Chem. Soc. 1995, 117, 5179-5197. 
(52) Birtwistle, D. T.; Herzenberg, A. J. Phys. B: At. Mol. Phys. 1971, $4,53-70$.

(53) Allan, M. J. Electron Spectrosc. Relat. Phenom. 1989, 48, 219-351.

(54) Janečková, R.; Kubala, D.; May, O.; Fedor, J.; Allan, M. Phys. Rev. Lett. 2013, 111, 213201.

(55) Li, X.; Bowen, K. H.; Haranczyk, M.; Bachorz, R. A.; Mazurkiewicz, K.; Rak, J.; Gutowski, M. J. Chem. Phys. 2007, 127, 174309. 